\section{Care staff training in detection of depression in residential homes for the elderly}

\author{
Randomised trial
}

\author{
A. M. H. EISSES, H. KLUITER, K. JONGENELIS, A. M. POT, A. T. F. BEEKMAN \\ and J. ORMEL
}

\author{
Background Many people with \\ depression in residential care homes for \\ the elderly do not receive treatment \\ because their depression remains \\ undetected.
}

\begin{abstract}
Aims To determine the effects of staff training on the detection, treatment and outcome of depression in residents of ten homes.
\end{abstract}

\begin{abstract}
Method We conducted a randomised controlled trial in ten residential homes. The intervention consisted of a training programme for staff and collaborative evaluation by staff and a mental health specialist of residents with possible depression.
\end{abstract}

\section{Results Recognition of depression} increased more in homes where staff received the training than in the control homes. Treatment rates also increased compared with control homes, but the increase was not significant. Residents with depressive symptoms had a more favourable course when staff had received training. Moreover, the prevalence of depressive symptoms decreased, but the decrease was not significant.

\section{Conclusions Training of care staff results in the increased detection of depression in the elderly, a trend towards more treatment and better outcomes.}

Declaration of interest None. Funding detailed in Acknowledgements.
Although depressive symptoms seriously affect the quality of life of a growing proportion of elderly people in residential care homes (Koenig \& Blazer, 1992; Ames, 1993; Blazer, 1994), many residents do not receive adequate antidepressant treatment (Rovner et al, 1991; Pond et al, 2002). Lack of recognition of depressive symptoms and signs by the attending staff in the residential home is a major obstacle to the provision of adequate treatment (Koenig et al, 1988; Rovner et al, 1991; Jackson \& Baldwin, 1993; Bagley et al, 2000). This study evaluates the effects of a programme of care staff training in residential homes on the recognition of depression, the treatment rate and the prognosis of those with depression. Our second aim was to study whether the training programme leads to lower prevalence and incidence rates for depression, since the training might positively affect the staff's attitude to those residents vulnerable to depression.

\section{METHOD}

\section{Design}

The study was a randomised controlled trial; ten residential care homes for the elderly were randomly assigned to either an experimental or a control group. The experimental group implemented a package of interventions. To compare the intervention with standard practice in residential homes, the control group provided care as usual. Several outcome measures were assessed because we evaluated the outcome of a whole care programme. The intervention was introduced in the month following baseline assessment. Research data were collected shortly before (baseline) and 6 months after the training by interviewers especially trained for this study. To guarantee an independent assessment, the interviewers were masked to the intervention and not associated with the residential homes. The researchers did not inform care staff of findings pertaining to the residents.

The main outcome measures referred to residents with depression (recognition, treatment and prognosis). The secondary outcome measure referred to the whole group of residents (prevalence). Written informed consent was obtained prior to the study. The medical ethics committee approved the study.

\section{Intervention}

The intervention was directed towards the care staff and consisted of two components: (a) training in using a standardised screening instrument; (b) review of the findings of screening at a staff meeting. The training focused on the recognition of psychopathology in residents and the recording of observations according to the Behaviour Rating Scale for Psychogeriatric Inpatients (Dutch abbreviation GIP-28). The GIP-28 consists of three reliable and valid scales: apathy, cognitive disturbance and affective disturbance (Jonghe et al, 1997). The GIP-28 has been used in the care of the elderly for over 15 years. During daily care activities the care staff observe specific behavioural aspects of residents. The staff later indicate how often they have registered certain behaviours. The advantage of this instrument is that active participation of the resident is not required. Trainers were mental health specialists; trainees were the care staff of the residential homes. The training consisted of two sessions of $2 \mathrm{~h}$. During the training, care staff learned how to observe specific behaviours, supported by video material. Special attention was given to the basic differences in behavioural manifestations of dementia and depression.

Evaluation of the intervention consisted of a discussion of the recorded observations by an experienced mental health nurse or psychologist with the care staff at a formal meeting to determine the course of action. Residents who were possibly depressed according to the GIP-28 were identified and discussed. The discussion resulted in a decision on the course of action:

(a) alerting staff to pay more attention to the resident;

(b) additional diagnostic assessment;

(c) referral to the general practitioner; or

(d) referral to a psychiatrist or mental health specialist. 
The effects of the intervention (e.g. successful detection) were assessed separately from the GIP recordings. Furthermore, no feedback was given - by the researchers or anyone else - to the care staff about the success of the detection of depression.

\section{Selection of homes}

In The Netherlands, residential homes provide daily care to the infirm elderly over 65 years with significant limitations to daily living; if needed, they also provide basic medical care. About $5 \%$ of all those over 65 years in The Netherlands live in a residential home. Nursing homes, in which about $3 \%$ of all Dutch residents over 65 years reside, provide more specialised medical care to all ages, but mainly to the elderly.

Of the 42 residential homes in the province of Drenthe, 23 were eligible for the study. The 19 non-eligible homes were excluded because they met one or more of the following exclusion criteria:

(a) ongoing or planned relocation, merger, changes in care methods or organisational instability (10 excluded);

(b) homes for specific populations (e.g. blind elderly) (3 excluded);

(c) participation in the pilot study or working with systematic screening procedures (6 excluded).

The staff of five of these homes had no interest in participation, as they received adequate assistance from the attending psychologist of a nearby nursing home. Five homes were not interested in the study, and three homes indicated that the intervention took too much time. Ultimately, ten homes were willing to participate.

Residential homes in the province of Drenthe are comparable to those in other parts of The Netherlands: they have the same gender ratio of residents (1 male: 4 female); mean residents' age (about 85 years); care methods; and admission criteria. However, homes in Drenthe are slightly smaller (about 85 beds) than those in other parts of the country (101 beds), and Drenthe itself consists of small towns (up to 150000 inhabitants) and rural areas (Centraal Bureau voor de Statistiek, 1998).

\section{Matching and randomisation}

Since the intensity of existing care might constitute a major confounder if not well balanced over both conditions, we matched the homes on care intensity. Care intensity was defined as: (a) the presence of contact nursing (one personal carer maintains intensive contact with the resident's family and general practitioner); and (b) care ratio (number of carers divided by the number of residents in the home). The matched homes were randomly assigned to the control or the experimental group. Control homes did not implement the intervention; they continued with standard care, comprising regular reports on residents by staff, without systematic observation or the use of rating scales.

\section{Selection of residents}

We visited all residents aged 65 years and above, except those receiving day care for dementia. The researchers notified residents of the study by a letter explaining the study and requesting their approval. Those who were severely cognitively impaired, indicated by a score below 15 on the Mini-Mental State Examination (MMSE) (Folstein et al, 1975), and those with severe hearing problems or aphasia were excluded. We reasoned that no valid assessments could be obtained from these residents.

\section{Assessments}

\section{Depression}

We assessed residents at baseline and follow-up with the validated Dutch version of the Geriatric Depression Scale (GDS), consisting of 30 yes/no items, which measures clinically relevant depressive symptoms (Yesavage et al, 1982; Kok, 1994). The GDS was administered at an interview between resident and trained research assistant, because many participants had serious difficulty with reading due to visual problems. The GDS does not contain any items assessing physical symptoms, hence it is an appropriate instrument for the elderly with physical illness. The GDS has been validated as a screening tool in nursing homes (McGivney et al, 1994). A score of over 10 on the GDS-30 is indicative of depression (Brink et al, 1982). Scores between 11 and 20 indicate moderate depression, and scores above 20 indicate severe depression (Brink et al, 1982).

\section{Recognition of depression}

Masked to the GDS results, the care staff were asked, at baseline and follow-up, to rate each resident as probably depressed or probably not depressed. The staff's ratings were compared with the scores of the residents on the GDS (above/below threshold). The GDS was the 'gold standard' in this study. Both sensitivity and specificity were calculated.

\section{Treatment of depression}

Treatment (yes/no) was defined as the prescription of antidepressant medication or counselling by a professional (e.g. general practitioner, psychologist or social worker). Interviews with care managers and medical records were used to obtain such information.

\section{Statistical analysis}

To determine prognosis, we compared residents from both experimental and control groups assessed as depressed (GDS $>10$ ) at baseline. To examine whether the intervention led to improvement of recognition, treatment and a lower prevalence of depression, data were required on all residents present at baseline and on all residents present at follow-up. Thus, for these analyses, 'new' residents were included at follow-up (new inhabitants, as well as those who were ill or refused participation at baseline).

Sensitivity refers to the proportion of residents with depression (GDS $>10$ ) correctly identified by the care staff. Specificity refers to the proportion of residents without depression (GDS $\leqslant 10)$ correctly identified by the care staff.

For the statistical evaluation of differences in proportions between experimental and control homes, taking into account baseline differences, Newcombe's method 10 for independent proportions was used (Newcombe, 2001). Differences in means were evaluated by $t$-tests. Where appropriate effect sizes were reported according to Cohen (1992).

\section{RESULTS}

\section{Baseline characteristics Homes}

Ten homes participated; five were assigned to the experimental group and five to the control group. The mean number of beds in each home was 75 (range 45-132) and did not differ between groups. The mean number of residents participating in each home was 41 (range 26-82). The ratio of care staff to residents, reflecting the intensity of care available, did not differ between the experimental group and the control group $(t$-test $=0.181, P=0.861)$. 
Table I Baseline characteristics of the sample of residents

\begin{tabular}{lll}
\hline Characteristic & $\begin{array}{l}\text { Control group } \\
(n=228)\end{array}$ & $\begin{array}{l}\text { Experimental group } \\
(n=198)\end{array}$ \\
\hline Demographic & & \\
Female residents: \% & 75.4 & 72.7 \\
Age in years: mean (s.d.) & $85.4(6.7)$ & $85.4(6.3)$ \\
MMSE score: mean (s.d.) & $23.1(3.9)$ & $23.2(4.2)$ \\
Length of stay (months) in residential & $34(1-245)$ & $25(1-235)$ \\
$\quad$ home at assessment: median (range) & & \\
Depression & & $5(0-25)$ \\
GDS scores: median (range) & $5(0-23)$ & $13.6(9.5-19.1)$ \\
GDS > I0: \% (95\% Cl) & $14.9(10.9-20.1)$ & \\
\hline
\end{tabular}

MMSE, Mini-Mental State Examination; GDS, Geriatric Depression Scale.

Table 2 Inclusion of residents at baseline and follow-up and reasons for attrition

\begin{tabular}{|c|c|c|}
\hline & $\begin{array}{c}\text { Experimental } \\
\text { group }\end{array}$ & $\begin{array}{l}\text { Control } \\
\text { group }\end{array}$ \\
\hline Eligible population & 263 & 276 \\
\hline \multicolumn{3}{|l|}{ Excluded } \\
\hline Deceased before assessment & $\mathrm{I}$ & 4 \\
\hline Refused to participate & 38 & 14 \\
\hline MMSE score too high & 18 & 23 \\
\hline Too ill or too deaf & 8 & 5 \\
\hline Not contacted & - & 2 \\
\hline Total excluded & 65 & 48 \\
\hline Included at baseline & 198 & 228 \\
\hline \multicolumn{3}{|l|}{ Attrition from baseline population } \\
\hline Deceased before follow-up-assessment & $28(14.1 \%)$ & $16(7.0 \%)$ \\
\hline Refusal & $29(14.6 \%)$ & $36(15.8 \%)$ \\
\hline MMSE score too high & 6 (3.0\%) & 9 (3.9\%) \\
\hline Too ill or too deaf & $6(3.0 \%)$ & $10(4.4 \%)$ \\
\hline Moved into nursing home & I (0.5\%) & $2(0.9 \%)$ \\
\hline Not contacted & 3 (1.5\%) & $0 \quad(0 \%)$ \\
\hline Total dropouts & 73 (36.9\%) & 73 (32.0\%) \\
\hline Available from baseline population for follow-up assessments & 125 & 155 \\
\hline Residents included from group that was excluded at baseline & 191 & $10^{2}$ \\
\hline Newly arrived residents included & 29 & 22 \\
\hline Total included at follow-up & 173 & 187 \\
\hline
\end{tabular}

MMSE, Mini-Mental State Examination.

I. Sixteen who refused participation at baseline and 3 were too ill at baseline.

2. Five who refused participation at baseline, 3 were too ill and 2 were not contacted at baseline.

\section{Residents}

There were 426 residents included at baseline; 41 residents were excluded because of severe cognitive impairment, 13 were physically too ill to participate, 5 had died shortly before the interviews and 2 residents could not be visited. There were 52 residents who refused to participate at baseline. Table 1 describes the baseline characteristics of the sample, divided into control and experimental
Table 2 shows the inclusion at baseline, the loss to follow-up and the inclusion of 'new' residents. Figure 1 presents a flow chart of inclusion and attrition rates at baseline and follow-up. At follow-up, data were available on 173 residents in the experimental group and 187 in the control group. Most residents were female $(76.9 \%)$. Of the men, $10.8 \%$ had a GDS score above $10 ; 11.9 \%$ of the women had a score above 10 .

In the experimental group 27 residents had depressive symptoms at baseline. Of these, 12 residents were also investigated at follow-up (15 were lost to follow-up: 7 refused, 5 died, 2 were too ill and 1 was too deaf). In the control group, 19 of the 34 GDS-positive residents at baseline participated at follow-up (15 were lost to follow-up: 3 died, 7 refused, 2 were too cognitively impaired and 3 were too ill).

Analyses of those who dropped out $(n=146)$ and those who were assessed twice $(n=280)$ revealed that the mean score on the GDS at baseline was significantly higher in those who dropped out $(7.29$, s.d. $=5.13)$ compared with those who were assessed twice $\quad(5.60, \quad$ s.d. $=4.24, \quad t$-test $=3.422$, $P=0.001)$. There were no age and gender differences between those who dropped out and those who were assessed twice. At follow-up, the mean GDS score of those assessed twice and new participants did not differ statistically $(6.07$, s.d. $=4.36 v$. 5.60 , s.d. $=4.45, t$-test $=-0.843, P=0.400$ ).

There was no difference in mean GDS score of those who dropped out from the control and experimental groups (7.22, s.d. $=5.24 v .7 .36$, s.d. $=5.05 ;$ t-test $=0.161$, $P=0.871$ ) or the mean score of those assessed twice in the two groups $(t$-test $=-1.008, \quad P=0.314)$. There was, however, a difference in mean scores of 'newcomers'. The newcomers in the control group had a higher mean GDS score (6.91, s.d.=4.895) than those in the experimental group $(4.73$, s.d. $=3.95, t$-test $=-2.102$, $P=0.040)$.

The mean GDS scores did not differ between the groups $(t$-test $=-0.458$, $P=0.647$ ). The average GDS scores at baseline did not differ among the ten homes (ANOVA $F=1.645, P=0.100$ ). 84.8 years (s.d. $=7.4$, range $65-98$ years) The mean age of the women was 85.6 years (s.d.=6.1, range 69-101 years). At baseline, $12.7 \%$ of the male residents suffered from depressive symptoms and $14.9 \%$ of the female residents. There were no significant baseline differences between the control and experimental groups.

\section{Care staff}

The sample of staff at baseline (42 in the control and 43 in the experimental group) included $10 \%$ nurses, $33 \%$ orderlies, $50 \%$ geriatric helpers and $7 \%$ others. These care 


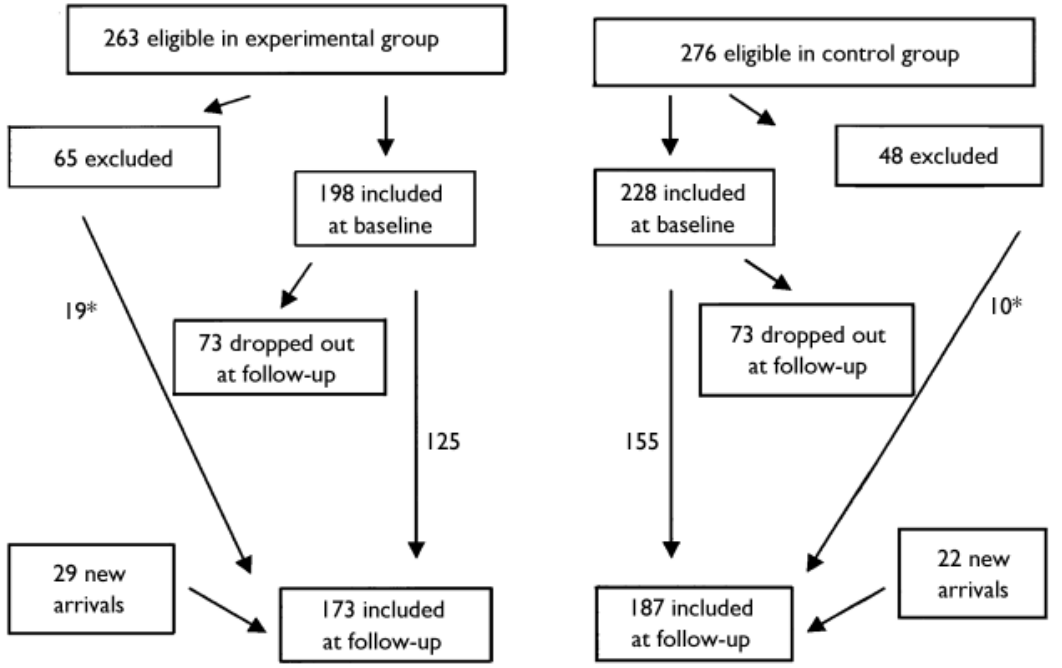

Fig. I Flow-chart of inclusion and attrition of respondents in the experimental and control groups at baseline and follow-up. *The number of respondents excluded at baseline because of illness, absence or refusal, but that did participate at follow-up.

Table 3 Recognition of depression in the experimental group before (baseline) and after training (follow-up) compared with the untrained control group (number of residents)'

\begin{tabular}{lccccc}
\hline & \multicolumn{2}{c}{ Baseline } & & \multicolumn{2}{c}{ Follow-up } \\
\cline { 2 - 3 } \cline { 5 - 6 } & Experimental & Control & & Experimental & Control \\
\hline Staff-/GDS - & 138 & 143 & & 137 & 128 \\
Staff+/GDS+ & 12 & 19 & & 8 & 13 \\
Staff+/GDS - & 30 & 39 & & 22 & 29 \\
Staff-/GDS+ & 15 & 11 & & 5 & 16 \\
Totals & 195 & 212 & & 172 & 186 \\
\hline
\end{tabular}

+, Depressed according to GDS > 10 or care staff; - , not depressed according to GDS $\leqslant 10$ or care staff.

I. Numbers differ slightly from Table 2 because of some missing staff (recognition) data.

staff members are the first who might notice depressive symptoms in residents and discuss their concern with colleagues.
All staff members except one were female. Their mean age was 37.7 years (s.d. $=7.5$, range 21-55) and they had worked on average 9.5 years in the homes under study (s.d. $=5$, range 10 months to 23 years, median $=10$ years). The samples are representative of the staff in Dutch homes for the elderly.

\section{Effects of intervention}

\section{Effect on recognition}

Table 3 shows the staff ratings (depressed/ not depressed) compared with the GDS scores (screen positive, GDS $>10 /$ screen negative, GDS $\leqslant 10)$. Table 4 shows the recognition rates (sensitivity and specificity). The improvement in sensitivity is significantly greater in the experimental group than in the control group where it actually decreased $(Z=1.6722$, $P=0.0472$ ).

\section{Effect on treatment for depression}

The treatment rate of residents with depressive symptoms showed a large difference at baseline in favour of the control group: $33.3 \%$ (11 out of 33 ) received treatment compared with $3.8 \%$ ( 1 out of 26 ) in the experimental group. The treatment rate in the experimental group increased (up to $23.1 \%, 3$ out of 13 ), but remained stable in the control group $(31 \%, 9$ out of 29$)$. Although substantial, the difference in increase of treatment rate was not statistically significant (Table 4).

\section{Effect on the course of depressive symptoms}

We defined the course of depression as favourable if the GDS score at follow-up fell into a less severe category than at baseline. In the experimental group, $58.3 \%$ (7 out of 12 ) improved $v .15 .8 \%$

Table 4 Sensitivity, specificity, positive predictive value, negative predictive value, treatment rate, improvement and prevalence of depressive symptoms (\%) in the experimental and control groups at baseline and follow-up

\begin{tabular}{|c|c|c|c|c|c|c|}
\hline & \multicolumn{2}{|c|}{ Experimental group } & \multicolumn{2}{|c|}{ Control group } & \multirow[t]{2}{*}{ Z-score' } & \multirow[t]{2}{*}{$P$ (one-sided) } \\
\hline & Baseline & Follow-up & Baseline & Follow-up & & \\
\hline Sensitivity & 44.4 & 61.5 & 63.3 & 44.8 & 1.6722 & 0.0472 \\
\hline Specificity & 82.1 & 86.2 & 78.6 & 81.5 & 0.1787 & 0.4291 \\
\hline Positive predictive value & 26.6 & 26.7 & 32.8 & 31.0 & -0.0069 & 0.4972 \\
\hline Negative predictive value & 90.2 & 96.5 & 92.9 & 88.9 & 2.3123 & 0.0104 \\
\hline Treatment rate & 3.8 & 23.1 & 33.3 & 31.0 & $\mathrm{I} .3677$ & 0.0857 \\
\hline Improvement of depressive symptoms & NA & 58.3 & NA & 15.8 & 2.4682 & 0.0068 \\
\hline Prevalence of depressive symptoms & 13.6 & 7.5 & 14.9 & 15.5 & -1.4022 & 0.0804 \\
\hline Incidence of depressive symptoms & NA & 3.5 & NA & 5.9 & -0.8593 & 0.1951 \\
\hline
\end{tabular}

NA, not applicable.

I. Z-scores of the difference in proportions between baseline and follow-up. The Z-score of 'improvement of depressive symptoms' refers to the difference between proportions in both conditions (Newcombe, 200I). 
(3 out of 19) in the control group ( $P=0.0068$, Table 4).

We also examined the decrease in depressive symptoms for participants with a positive GDS score at baseline (GDS $>10$ ). In the experimental group the GDS decreased by a mean of -4.50 GDS units $($ s.d.=4.76) but increased by 0.684 GDS units $($ s.d. $=4.12)$ in the control group. The corresponding effect size was $-1.18(95 \%$ CI -1.93 to -0.38 ) suggesting that the GDS scores of residents who were depressed at baseline (GDS >10) decreased significantly more in the experimental than in the control group.

\section{Effect on the prevalence and incidence of depression}

We also investigated whether the whole sample benefited from the intervention. The prevalence of depressive symptoms (GDS >10) at baseline was similar in both groups, with $13.6 \%$ in the experimental group and $14.9 \%$ in the control group. At follow-up, the prevalence of depressive symptoms in the experimental group decreased to $7.5 \%$ but remained at $15.5 \%$ in the control group. Although substantial, the difference between the groups was not significant (Table 4). We also compared the decrease in depressive symptoms between baseline and follow-up. The mean difference score was 0.1360 (s.d.=3.4) in the experimental group and 0.7419 (s.d.=3.2) in the control group. The effect size was $-0.18(95 \% \mathrm{CI}-0.42$ to 0.005$)$ (NS).

Moreover, the incidence of depressive symptoms (GDS $>10$ ) at follow-up in residents without depression at baseline was $3.5 \%$ in the experimental group and $5.9 \%$ in the control group. There is a suggestion that the intervention contributes to the prevention of depressive symptoms, but the difference was not statistically significant $(Z=-0.8593, P=0.1951)$.

\section{DISCUSSION}

\section{Methodological considerations}

In the current study, a self-rating instrument of depression was used instead of a diagnostic interview. This may have included false positives, but 'adequate diagnosis of depression by care staff' was not the goal of the intervention. We were interested in the education of care staff in the recognition of those residents apparently suffering from depressive symptoms.
The two groups of care homes were well-balanced with respect to degree of care. However, we did not succeed in creating two equivalent groups at baseline with regard to recognition and treatment rates. Although we have carefully checked the procedures, we can not explain the difference. We are reassured that the baseline difference was coincidental, but would have preferred all baseline indices to be (roughly) equivalent. With ten homes there is a considerable probability of baseline inequality. In this type of intervention the number of randomisable units is by implication always lower than one would wish from a statistical and a design point of view. Unlike many studies at the institutional level we applied baseline assessments; by doing this we were able to correct for baseline non-equivalence and calculate the change in scores brought about by the intervention.

The study had high rates of loss to follow-up of residents with depression. Hence, only small numbers of residents with depression were available for analyses of improvement. The loss to follow-up reflects the vulnerability of residents with depression.

Newcomers in the control group were more depressed than those in the experimental group, but there was no overall difference in symptom rates between new participants and residents assessed twice. This may be a result of support of newly arrived residents by the care staff. An attentive and supporting attitude may be enhanced by training.

The effects we found were not large. This is because the number of residents with depression in our study was much smaller than expected from previous prevalence studies. Such a small number restricts the maximum effect attainable, through the phenomenon of 'restriction of range'. The intervention may be more effective in populations with higher prevalences (Nunnally, 1976).

Furthermore, the sensitivity decreased in the control group at follow-up, probably because of a reduction in awareness or demoralisation owing to not having received the training. These phenomena are documented in the literature on research methodology of intervention studies (Cook \& Campbell, 1979).

\section{Effect on recognition}

Recognition of depression in the elderly in residential homes is undoubtedly difficult; difficulties result from the high prevalence of multiple physical disorders and functional impairments in residents (Koenig et al, 1993). The intervention under study brought about an increase in the recognition rates (sensitivity). At the same time, the specificity remained high and stable, implying that the care staff improved their recognition of depression, without wrongly rating nondepressed residents as depressed. Furthermore, the positive predictive value of the judgements of care staff remained stable in both groups between baseline and follow-up, whereas the negative predictive value increased after the intervention.

Judgements of care staff are without doubt valuable in the recognition of depression, but before psychological or pharmacological treatment for the depression may be provided, screening instruments and clinical assessments by, for example, general practitioners are still mandatory.

\section{Effect on treatment for depression}

The treatment rate of residents with depressive symptoms increased after the intervention. The increase was substantial but not statistically significant. This supports the results of another recent randomised controlled trial carried out in long-term care facilities: the frequency of treatment or referral to mental health services by primary care physicians increased when they were informed about the results of a depression screen (GDS) (Soon \& Levine, 2002).

\section{Effect on the course of depressive symptoms}

Residents with depressive symptoms improved more in the homes where the intervention had been implemented than in the control homes. Our results are in line with those reported by Cuijpers \& van Lammeren (2001), who applied a quasiexperimental design. They reported favourable patient outcome as a result of a comprehensive training programme in residential homes focusing on caregivers, residents and relatives. Beneficial effects of training and education of care staff on the course of depressive symptoms have also been reported by Proctor and colleagues (1999). Rabins et al (2000) also found positive effects of an intervention on the reduction of psychiatric symptoms in the elderly. They taught staff to find cases, to perform assessment in the residents' apartments and to provide care if necessary. This 
method compares well with that used in our study.

\section{Effect on the prevalence and incidence of depression}

Our findings suggest that the intervention contributes to the prevention of depressive symptoms, since in the experimental group: (a) the prevalence rates of depressive symptoms showed a greater decrease between baseline and follow-up (NS); and (b) the incidence of depressive symptoms was lower compared with the control group (NS).

In summary, we have found support for the beneficial effects of a programme of staff training in improving detection, treatment and the course of depression in normal practice. The care staff appreciated the training, the systematic observation procedures and the meetings with the mental health worker. They indicated that they received valuable tools to deal with vulnerable residents. The intervention has now been implemented successfully in several parts of The Netherlands.

\section{ACKNOWLEDGEMENTS}

This study is based on data collected in the context of the Amsterdam Groningen Elderly Depression (AGED) study, conducted at the Department of Nursing Home Medicine and Psychiatry and the Institute of Extramural Medicine (EMGO), VU University Medical Center in Amsterdam and the Department of Social Psychiatry, University Medical Center in Groningen. The study is primarily funded by The Netherlands Organisation of Scientific Research, Programme Chronic Disease (940-3304I). We gratefully acknowledge the cooperation of the staff and residents of the residential homes, and the GGZ-Drenthe, the regional mental health institution, that carried out the intervention programme.

\section{REFERENCES}

Ames, D. (1993) Depressive disorders among elderly people in long-term institutional care. Australian and New Zealand Journal of Psychiatry, 27, 379-391.

\section{Bagley, H., Cordingley, L., Burns, A., et al (2000)} Recognition of depression by staff in nursing and residential homes. Journal of Clinical Nursing, 9, 445-450.

Blazer, D. G. (1994) Epidemiology of depression: prevalence and incidence. In Principles and Practice of Geriatric Psychiatry (eds J. R. M. Copeland, M. T. AbouSaleh \& D. G. Blazer), pp. 519-522. Chichester: Wiley.

Brink, T. L., Yesavage, J. A., Lum, O., et al (1982) Screening tests for geriatric depression. Clinical Gerontologist, I, 37-49.

Centraal Bureau voor de Statistiek (1998) Bevolking der gemeenten van Nederland op I januari 1998. Voorburg/Heerlen, The Netherlands: Centraal Bureau voor de Statistiek.

Cohen, J. (1992) A power primer. Psychological Bulletin, II2, 155-159.

Cook, T. D. \& Campbell, D. T. (1979) Quasi-

Experimentation: Design and Analysis for Field Settings.

Chicago, IL: Rand McNally.

\section{CLINICAL IMPLICATIONS}

- Training care staff in systematic observation contributed to the improvement of detection, treatment and course of depressive symptoms in the elderly living in residential homes.

- Improvement of sensitivity was achieved with high stable specificity. Hence, unnecessary treatment of non-depressed residents was avoided.

- Focused involvement of care staff in detecting mental health problems is feasible, desirable and worthwhile.

\section{LIMITATIONS}

- Despite matching and randomisation, the experimental and control groups had unequal starting points with regard to recognition and treatment rates.

- A self-rating instrument of depression was used instead of a diagnostic interview.

- The study had restricted numbers of residents with depressive symptoms which limits the maximum effects achievable.

A. M. H. EISSES, MSc, H. KLUITER, PhD, Department of Psychiatry, Medical Faculty, University of Groningen; K. JONGENELIS, MD, A. M. POT, PhD, A. T. F. BEEKMAN, MD, PhD, EMGO Institute, Free University Amsterdam; J. ORMEL, PhD, Department of Psychiatry, Medical Faculty, University of Groningen, The Netherlands

Correspondence: Ms Anne-Marie Eisses, Victorialaan 7, 5261 AE Vught, The Netherlands. Tel: +31 736577422; Fax: +31 503619722; e-mail: A.M.H.Eisses@med.rug.nl

(First received 24 February 2004, final revision I November 2004, accepted 5 November 2004)

Cuijpers, P. \& van Lammeren, P. (200I) Secondary prevention of depressive symptoms in elderly inhabitants of residential homes. International journal of Geriatric Psychiatry, 16, 702-708.

Folstein, M. F., Folstein, S. E. \& McHugh, P. R. (1975) 'Mini-mental state'. A practical method for grading the cognitive state of patients for the clinician. Journal of Psychiatric Research, 12, 189-198.

Jackson, R. \& Baldwin, B. (1993) Detecting depression in elderly medically ill patients: the use of the Geriatric Depression Scale compared with medical and nursing observations. Age and Ageing, 22, 349-353.

Jonghe, J. F. M. de, Ooms, M. E. \& Ribbe, M. W. (1997) Verkorte Gedragsobservatieschaal voor de Intramurale Psychogeriatrie (GIP-28). Tijdschrift voor Gerontologie en Geriatrie, 28, 119-123.

Koenig, H. G. \& Blazer, D. G. (1992) Epidemiology of geriatric affective disorders. Clinics in Geriatric Medicine, 8, 235-251.

Koenig, H. G., Meador, K. G., Cohen, H. J., et al (1988) Detection and treatment of major depression in older medically ill hospitalized patients. International journal of Psychiatry In Medicine, 18, 17-31.

Koenig, H. G., Cohen, H. J., Blazer, D. G., et al (1993) Profile of depressive symptoms in younger and older medical inpatients with major depression. Journal of the American Geriatric Society, 4I, II69-1176.

Kok, R. M. (1994) Zelfbeoordelingsschalen voor depressie bij ouderen. Tijdschrift voor Gerontologie en Geriatrie, 25, 150-155.

McGivney, S. A., Mulvihill, M. \& Taylor, B. (1994) Validating the GDS depression screen in the nursing home. Journal of the American Geriatric Society, 42 , 490-492.

Newcombe, R. G. (200I) Estimating the difference between differences: measurement of additive scale interaction for proportions. Statistics in Medicine, 20 2885-2893.

Nunnally, J. C. (1976) Psychometric Theory. New York McGraw-Hill.

Pond, C. D., Mant, A., Bridges-Webb, C., et al (2002) Recognition of depression in the elderly: a comparison of general practitioner opinions and the geriatric depression scale. Family Practice, 7, 190-194.

Proctor, R., Burns, A., Stratton Powell, H., et al (1999) Behavioural management in nursing and residential homes: a randomised controlled trial. Lancet, 354, 26-29.

Rabins, P. V., Black, B. S., Roca, R., et al (2000) Effectiveness of a nurse-based outreach program for identifying and treating psychiatric illness in the elderly JAMA, 283, 2802-2809.

Rovner, B. W., German, P. S., Brant, L. J., et al (1991) Depression and mortality in nursing homes. JAMA, 265 993-996.

Soon, J. A. \& Levine, M. (2002) Screening for depression in patients in long-term care facilities: a randomized controlled trial of physician response. Journal of the American Geriatrics Society, 50, 1092-1099.

Yesavage, J. A., Brink, T. L., Rose, T. L., et al (1982) Development and validation of a geriatric depression screening scale: a preliminary report. Journal of Psychiatric Research, 17, 37-49. 